\title{
Complications of systemic therapy - gut infections and acute diarrhoea
}

\author{
Authors: Daniel LY Lee ${ }^{A}$ and Alan Anthoney ${ }^{B}$
}

\section{Background}

Diarrhoea forms part of the symptom burden of many patients with cancer and can manifest from a low-grade but persistent annoyance to a life-threatening toxicity requiring inpatient admission and urgent medical care. Acute and chronic forms of diarrhoea are differentiated by their duration (acute diarrhoea lasting for up to 4 weeks). ${ }^{1}$ Acute diarrhoea is a common adverse effect of systemic anticancer therapy (SACT) affecting up to $80 \%$ of patients treated with some chemotherapy regimens. ${ }^{2}$ Diarrhoea grading is described in Table 1 ; however, the patient's perception of the burden of their toxicity might differ from the classical medical description of its severity. ${ }^{3}$

Acute diarrhoea in patients receiving treatment for cancer can arise as a direct consequence of damage to the bowel from chemotherapy or radiotherapy or indirectly (eg from secondary infection or the effects of previous surgery).

Distinguishing between these differing aetiologies for diarrhoea in patients receiving cancer treatment is important for determining appropriate management. Previous surgery to the gastrointestinal tract can result in altered gastric emptying or bile salt flow, bacterial overgrowth or hepatic and/or pancreatic insufficiency; all can result in diarrhoea that can be exacerbated by systemic anticancer treatment. Overflow diarrhoea associated with bowel stasis and incomplete obstruction can result directly from the effects of the cancer itself or as a consequence of medications (eg opiates), abdominal surgery or radiotherapy (eg pelvic radiotherapy having effects on loops of small bowel or the sigmoid colon). ${ }^{4-6}$

\section{Assessment and management}

The management of diarrhoea in patients with cancer receiving SACT can be markedly different compared with patients on a general medical ward, because antidiarrhoeals might need to be used early and aggressively, before stool culture results are available. Fig 1 outlines a common assessment and treatment pathway for patients on chemotherapy with diarrhoea. The most important distinction to make when assessing a patient is whether the

Authors: AST6 medical oncology, Huddersfield Royal Infirmary, Huddersfield, UK; BConsultant in medical oncology, St James's Institute of Oncology, Leeds, UK diarrhoea is secondary to either infection or to treatment. Guides to an infective cause for assessing acute diarrhoea are symptoms such as fever, contact with a person with diarrhoea, exposure to possible infective sources (eg eating out) or recent travel abroad. ${ }^{7}$

\section{Gut infection}

Cytotoxic chemotherapy agents directly affect the gastrointestinal mucosa and cause inflammation, oedema, ulceration and atrophy, leading to increased permeability of the bowel mucosa and increased susceptibility to the spread of organisms from the intestine across the bowel wall into the blood stream. This can result in septicaemia and shock, especially if it coincides with chemotherapy-induced neutropenia..$^{4,8,9}$ The gastrointestinal symptoms caused through infection can respond quickly to antibiotics. The work up of such patients should be timely because appropriate early intervention can decrease morbidity and mortality.

Although coliforms, such as Escherichia coli, are the most common pathogens causing sepsis in patients with chemotherapy-induced diarrhoea (CID), other organisms, such as Clostridium difficile, fungal infections and viruses, can be responsible for severe symptoms. Clostridium difficile might not be associated with previous antibiotic use in patients on chemotherapy and can present without typical features; for example, $10 \%$ of cases are toxin negative and, in neuropenic patients, the typical pseudomembranes seen on endoscopy might not be obvious. Treatment includes oral metronidazole or vancomycin, depending on local protocol and severity. In patients with severe or prolonged treatment-induced immunosuppression, infection with viral pathogens, such as cytomegalovirus (CMV), should be considered. Empirical treatment should be considered when there is a high index of suspicion because positive detection of CMV (by culture or polymerase chain reaction) can take several weeks. ${ }^{4}$

\section{Acute diarrhoea secondary to anticancer treatment}

Chemotherapy-induced diarrhoea (CID) can occur in up to $80 \%$ of patients, depending on the regimen. Combinations of agents can have additive or synergistic effects with greater frequency and severity of diarrhoea (Table 2). Agents involved in treating cancers of the gastrointestinal tract are those most commonly associated with severe diarrhoea.

There are several specific factors that can influence the likelihood of CID with individual chemotherapy agents. 


\section{Table 1. Common terminology criteria for adverse events (v4.0).}

\begin{tabular}{|c|c|c|c|c|}
\hline Grade & 1 & 2 & 3 & 4 \\
\hline Colitis & $\begin{array}{l}\text { Asymptomatic; clinical or } \\
\text { diagnostic observation only; } \\
\text { intervention not indicated }\end{array}$ & $\begin{array}{l}\text { Abdominal pain; mucous } \\
\text { or blood in stool }\end{array}$ & $\begin{array}{l}\text { Severe abdominal pain; change in } \\
\text { bowel habits; medical intervention } \\
\text { indicated; peritoneal signs }\end{array}$ & $\begin{array}{l}\text { Life-threatening } \\
\text { consequences; urgent } \\
\text { intervention indicated }\end{array}$ \\
\hline Diarrhoea & $\begin{array}{l}\text { Increase of }<4 \text { stools a day } \\
\text { over baseline; mild increase } \\
\text { in ostomy output compared } \\
\text { with baseline }\end{array}$ & $\begin{array}{l}\text { Increase of } 4-6 \text { stools a } \\
\text { day over baseline; } \\
\text { moderate increase in } \\
\text { ostomy output compared } \\
\text { with baseline }\end{array}$ & $\begin{array}{l}\text { Increase of }>7 \text { stools a day over } \\
\text { baseline; incontinence; hospitalisation } \\
\text { indicated; severe increase in ostomy } \\
\text { output compared with baseline; limiting } \\
\text { self-care activities of daily living }\end{array}$ & $\begin{array}{l}\text { Life-threatening } \\
\text { consequences; urgent } \\
\text { intervention indicated }\end{array}$ \\
\hline
\end{tabular}

\section{Oral anticancer treatments}

With orally administered chemotherapy, patients are ultimately responsible for taking the medication. Some patients, in the hope of increasing treatment effectiveness, will continue to take their tablets despite the onset of severe and increasing toxicity. Good patient selection and education are vital and can reduce the morbidity associated with this treatment route. ${ }^{10}$

\section{Fluorouracil and dihydropyrimidine dehydrogenase}

Up to $5 \%$ of patients have genetic polymorphisms resulting in reduced activity of dihydropyrimidine dehydrogenase
Fig 1. Flow diagram for the management of patients with diarrhoea while receiving chemotherapy. Common terminology criteria grading is given in Table 1. CDT = Clostridium difficile toxin; $F B C=$ full blood count; Mag = magnesium;

LFTs = liver function tests;

$\mathrm{U} \& \mathrm{E}=$ urea and electrolytes.
Diarrhoea

While receiving chemotherapy

$\downarrow$

History and examination: Are adverse features present?

For example - neutropenia, blood or mucus in stool, fever, dehydration, vomiting, poorly controlled comorbidities

(eg diabetes).

Investigations: Blood test (FBC, U\&E, Mag, LFT) and stool samples including CDT.

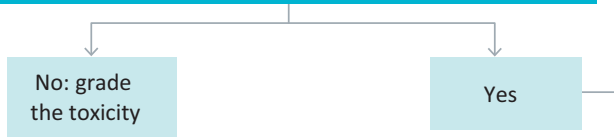

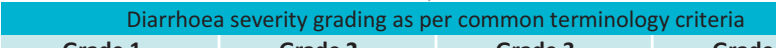

Grade 1

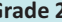

rade 3

Grade 4

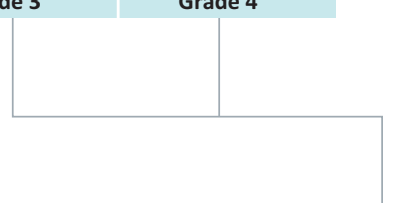

mg dose loperamide

after each loose stool; maximum

$16 \mathrm{mg}$ day)

Diarrhoea resolved:
Continue loperamide
for 12 hours after
resolution and
normal diet has been
resumed
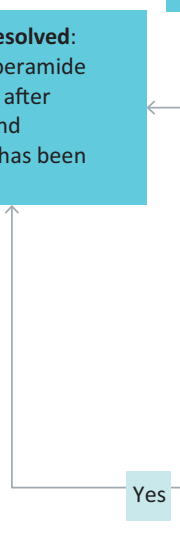

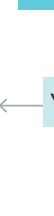

\section{day)}

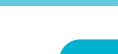

Resolution

after 24

hours

\section{No}

Diarrhoea not resolved:

High-dose loperamide

(4 mg after first stool

then $2 \mathrm{mg}$ every 2 hours)

Resolution

Yes

after 24

hours
Admit to hospital: Commence intravenous fluid and electrolyte replacement; commence octreotide 100-150 mcg s/cut daily; test stool samples including for CDT; consider antibiotic cover 


\begin{tabular}{|c|c|}
\hline Chemotherapy regimen & Diarrhoea: Grade 3/4 \\
\hline 5 fluorouracil (5FU) - bolus & $32 \%$ \\
\hline Irinotecan & $20 \%$ \\
\hline Capecitabine & $10-15 \%$ \\
\hline Capecitabine + irinotecan & $21.5 \%$ \\
\hline Oxaliplatin + 5FU & $6 \%$ \\
\hline Oxaliplatin + capecitabine & $18 \%$ \\
\hline Docetaxel & $4 \%$ \\
\hline Docetaxel + capecitabine & $14 \%$ \\
\hline
\end{tabular}

(DPD), an enzyme central to fluorouracil catabolism. Administration of a standard dose of 5 fluorouracil (5FU) in such patients results in significantly prolonged exposure to higher concentrations of the drug, resulting in early onset and increased severity of diarrhoea and other toxicities. Patients should be managed aggressively as above and further 5FU use would not be recommended. ${ }^{12}$

\section{Irinotecan}

Irinotecan is associated with two distinct forms of diarrhoea. Early-onset diarrhoea (within $24 \mathrm{~h}$ ) is associated with an acute cholinergic reaction and often with rhinitis and salivation. This should be treated with atropine and usually resolves quickly. Late-onset diarrhoea is particularly difficult; it is associated with the production of irinotecan metabolites within the gastrointestinal tract and can produce profuse diarrhoea. It tends to occur around 7-10 days post treatment, corresponding

\section{Key points}

Diarrhoea is a common and important symptom in patients receiving chemotherapy

The aetiology of diarrhoea can relate to the cancer treatment (chemotherapy, radiotherapy or surgery) or be infective in nature

Antidiarrhoeals might be indicated much earlier than usual, especially if the diarrhoea is secondary to treatment rather than infection

Some patients might be more prone to toxicity, including diarrhoea, experiencing diarrhoea earlier and with greater severity than usually expected, for example in dihydropyrimidine dehydrogenase deficiency

New biological agents, such as ipilimumab, can result in diarrhoea through different mechanisms of action and require specialist oncology advice and management from early on

KEYWORDS: Diarrhoea, chemotherapy, infection, management, neutropenia with the most likely time for neutropenia. Ciprofloxacin is often given to patients if moderate diarrhoea develops at home, to cover for Gram-negative sepsis. ${ }^{9}$

Polymorphisms in the UGT1A1 gene (whose protein product is involved in irinotecan degradation) have been associated with an increased risk of severe diarrhoea with moderate or high doses of irinotecan.

\section{Biological treatment}

Diarrhoea is a common adverse effect of the newer targeted anticancer therapies and occurs in up to $60 \%$ of patients. Drug interactions (between different targeted therapies or in combination with classical cytotoxics) can increase the risk and severity of diarrhoea. Particularly important or common drugs are listed in Table 3.

Ipilimumab, used in patients with malignant melanoma, acts differently compared with other commonly used biological therapies by affecting the immune response of the body to cancer cells through modulating $\mathrm{T}$ cell immunity. Ipilimumab-associated diarrhoea (up to $40 \%$ of treated patients) is an immune-related adverse effect. In approximately $7 \%$ of patients, the diarrhoea is Grade 3 or more and can occasionally be life threatening. ${ }^{13}$ Concern is raised if diarrhoea is associated with other gastrointestinal toxicities, such as nausea, vomiting or cramping abdominal pain, or the presence of blood in the diarrhoea. Low-grade persistent diarrhoea beyond 7 days is also a concern. These patients should be treated in close liaison with the oncology team to discuss possible endoscopy, corticosteroid or infliximab therapy. ${ }^{14}$

\section{Chemoradiotherapy}

Patients receiving concurrent chemotherapy and radiotherapy (chemoradiotherapy) can be more susceptible to diarrhoea, particular if receiving radiotherapy treatment to the pelvis. ${ }^{5}$ Peak

Table 3. Rates of diarrhoea in commonly used biological anticancer agents.

Drug

\begin{tabular}{lll} 
& \multicolumn{2}{c}{ Diarrhoea } \\
\hline Any & Grade & Special consideration \\
Grade & $3 / 4$
\end{tabular}

Erlotinib (non-small $\quad 54 \% \quad 6 \%$ cell lung cancer)

Lapatinib in $\quad 65 \%$ Rare Responds quickly to
combination with antidiarrhoeals capecitabine $\left(1,000 \mathrm{mg} / \mathrm{m}^{2}\right)$

$\begin{array}{lll}\text { Sunitinib } & 52.3 \% & 5.9 \% \\ \text { Bevacizumab } & 21 \% & 1 \%\end{array}$

Low association with gastrointestinal fistulae and perforation

Ipilimumab $40 \%$ $6.9 \%$
Occasionally fatal refer to protocol for dose delay and adjuvants 
incidence for acute diarrhoea is 1-2 weeks after radiotherapy, when the toxicity to the gastrointestinal mucosa is at its highest. This needs to be proactively treated to reduce the burden of toxicity in patients, as described by Wang-Gillam for the treatment of patients with pancreatic cancer with chemoradiotherapy. ${ }^{15}$

There is increasing recognition of the long-term gastrointestinal toxicity as a result of radiotherapy. Specialist services and support are available for those with long-term toxicity and its sequelae (eg malabsorption and rectal bleeding).

\section{Neutropenia and typhlitis}

Typhlitis is bowel inflammation usually involving the caecum in a patient with chemotherapy-induced neutropenia. It is associated with a triad of right lower quadrant pain, fever and bloody diarrhoea. As any part of the bowel can become involved with some chemotherapies, particularly with taxane use or drug combinations used in haematological malignancies, the term 'neutropenic enterocolitis' (NEC) has gained popularity. ${ }^{16}$

In either case, the patient should receive treatment similarly to any other neutropenic patient alongside aggressive antidiarrhoea management, including aggressive fluid resuscitation and broad-spectrum antibiotics, antifungals can also be indicated. NEC is associated with a gastrointestinal tract thickness of $>4 \mathrm{~mm}$ on imaging.

There is a high mortality associated with this rare condition. Complications include bacteraemia, haemorrhage and bowel wall perforation or abscess formation. If the bleeding is severe, surgical intervention might be necessary if appropriate. ${ }^{16-18}$

\section{Adjuvants to treatment}

Supportive medications, such as antibiotics, antiemetics, laxatives and some herbal remedies, alongside systemic treatment can also lead to diarrhoea. These should be considered together with a general medical and medicines review.

\section{Future developments in managing chemotherapy- induced diarrhoea}

Identification of individual patients at greater risk of CID would enable treatment modifications in advance. It is known that gene polymorphisms for enzymes involved in the metabolism of cytotoxic chemotherapy agents can result in increased drug exposure causing greater likelihood and severity of CID. Assays to detect possible enzyme deficiency states are available (some commercially) or under development; for example, DPD assays for 5FU toxicity and UGT1A1 assays for irinotecan toxicity. However, evidence supporting the predictive benefits of such assays remains contradictory.

Prophylactic measures to reduce the incidence or severity of CID have been researched, with multiple trials trying to identify the optimum prophylactic measure for CID. Probiotics have produced mixed results in diarrhoea of all causes, but use in patients receiving chemotherapy needs further study. Long-acting octreotide has been used, without clinical trial, for patients with rectal cancer at high risk of CID and in those with severe diarrhoea but whom dose reduction is undesirable. ${ }^{2}$ Slow-release steroids (eg budesonide), which become active within the small and large intestine, are used in inflammatory bowel disease and have been trialled in CID. ${ }^{9}$

\section{Conclusion}

Diarrhoea is a common and important toxicity. Its management differs from that within other medical specialties and early and aggressive use of antidiarrhoeals is paramount. Good patient education and support, together with prompt medical treatment, can reduce both morbidity and mortality.

\section{References}

1 Tidy C. Acute diarrhoea in adults, 2012. www.patient.co.uk/doctor/ acute-diarrhoea-in-adults-pro. [Accessed 4 August 2014].

2 Uronis HE, Bendell JC. Anal cancer: an overview. Oncologist 2007;12:524-34.

3 Carlotto A, Hogsett VL, Maiorini EM et al. The economic burden of toxicities associated with cancer treatment: review of the literature and analysis of nausea, vomiting, diarrhoea, oral mucositis and fatigue. Pharmacoeconomics 2013;31:753-66.

4 Andreyev HJV, Davidson S, Gillespie C et al. Practice guidance on the management of acute and chronic gastrointestinal problems arising as a result of treatment for cancer. Gut 2012;61:179-92.

5 Muls AC. Gastrointestinal consequences of cancer treatment and the wider context: a bad gut feeling. Acta Oncol 2014;53:297-306.

6 Pergolizzi S, Maranzano E, DeAngelis V et al. Diarrhoea in irradiated patients: a prospective multicentre observational study. Dig Liver Dis 2013;45:933-7.

7 National Institute for Health and Care Excellence. Diarrhoea adults' assessment, 2013. http://cks.nice.org.uk/diarrhoea-adultsassessment\#!topicsummary [Accessed 4 August 2014].

8 Maroun JA, Anthony LB, Blais N et al. Prevention and management of chemotherapy-induced diarrhoea in patients with colorectal cancer: a consensus statement by the Canadian Working Group on Chemotherapy-Induced Diarrhoea. Curr Oncol 2007;14:13-20.

9 Stein A. Chemotherapy-induced diarrhoea: pathophysiology, frequency and guideline-based management. Ther Adv Med Oncol 2010;2:51-63.

10 Moore S. Facilitating oral chemotherapy treatment and compliance through patient/family-focused education. Cancer Nursing 2007; 30:112-22.

11 Lee DLY, Anthoney DAA. Gut infections and acute diarrhoea. In: Marshall E, Young A, Clark P, Selby P (eds), Problem solving in acute oncology. Oxford: Clinical Publishing, 2014;120-6.

12 Cancer Research UK. DPD deficiency and fluorouracil, 2013. www. cancerresearchuk.org/cancer-help/about-cancer/cancer-questions/ dpd-deficiency-and-fluorouracil [Accessed 4 August 2014].

13 Sznol M, Callahan MK, Yuan J, Wolchok J. Key issues in the management of gastrointestinal immune-related adverse events associated with ipilimumab administration. J Commun Support Oncol 2013;10:351-8.

14 Weber JS, Kahler KC, Hauschild A. Management of immune-related adverse events and kinetics of response with ipilimumab. J Clin Oncol 2012;30:2691-7.

15 Wang-Gillam A, Abrams RA, Posner MC et al. Supportive care considerations during concurrent chemoradiotherapy for pancreatic adenocarcinoma: lessons learned from clinical experience. Am J Clin Oncol 2013;36:637-43.

16 Nesher L, Rolston KV. Neutropenic enterocolitis, a growing concern in the era of widespread use of aggressive chemotherapy. Clin Infect Dis 2013;56:711-7.

17 Davila ML. Neutropenic enterocolitis. Curr Opin Gastroenterol. 2006;22:44.

18 Bremer CT, Monahan BP. Necrotizing enterocolitis in neutropenia and chemotherapy: a clinical update and old lessons relearned. Curr Gastroenterol Rep 2006;8:333.

Address for correspondence: Dr A Anthoney, St James's Institute of Oncology, Beckett Street, Leeds LS9 7TF.

Email: D.A.Anthoney@leeds.ac.uk 